\title{
X-RAY ANALYSIS OF THE PROPER MOTION AND PULSAR WIND NEBULA FOR PSR J1741-2054
}

\author{
Katie Auchettl ${ }^{1,2}$, Patrick Slane ${ }^{1}$, Roger W. Romani ${ }^{3}$, Bettina Posselt ${ }^{4}$, George G. Pavlov ${ }^{4}$, Oleg Kargaltsev ${ }^{5}$, \\ C-Y. NG ${ }^{6}$, Tea Temim ${ }^{7,8}$, Martin. C. Weisskopf ${ }^{9}$, Andrei Bykov ${ }^{10}$, and Douglas A. Swartz ${ }^{9}$ \\ ${ }^{1}$ Harvard-Smithsonian Center for Astrophysics, 60 Garden Street, Cambridge, MA 02138, USA \\ ${ }^{2}$ School of Physics \& Astronomy, Monash University, Melbourne, Victoria 3800, Australia \\ ${ }^{3}$ Department of Physics, Stanford University, Stanford, CA 94305, USA \\ ${ }^{4}$ Department of Astronomy \& Astrophysics, Pennsylvania State University, 525 Davey Lab, University Park, PA 16802, USA \\ ${ }^{5}$ Department of Physics, The George Washington University, 72521 st St, NW, Washington, DC 20052, USA \\ ${ }^{6}$ Department of Physics, The University of Hong Kong, Pokfulam Road, Hong Kong, China \\ ${ }^{7}$ Observational Cosmology Lab, Code 665, NASA Goddard Space Flight Center, Greenbelt, MD 20771, USA \\ ${ }^{8}$ CRESST, University of Maryland-College Park, College Park, MD 20742, USA
${ }^{9}$ NASA/Marshall Space Flight Center, ZP12, 320 Sparkman Drive, Huntsville, AL 35805, USA \\ ${ }^{10}$ A.F. Ioffe Physical-Technical Institute, St. Petersburg 194021, also St.Petersburg State Politechnical University, Russia \\ Received 2014 December 22; accepted 2015 February 20; published 2015 March 24
}

\begin{abstract}
We obtained six observations of PSR J1741-2054 using the Chandra ACIS-S detector totaling $~ 300 \mathrm{ks}$. By registering this new epoch of observations to an archival observation taken $3.2 \mathrm{yr}$ earlier using X-ray point sources

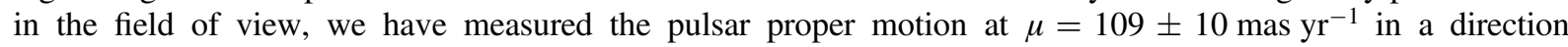
consistent with the symmetry axis of the observed $\mathrm{H} \alpha$ nebula. We investigated the inferred past trajectory of the pulsar but find no compelling association with OB associations in which the progenitor may have originated. We confirm previous measurements of the pulsar spectrum as an absorbed power law with photon index $\Gamma=2.68 \pm 0.04$, plus a blackbody with an emission radius of $\left(4.5_{-2.5}^{+3.2}\right) d_{0.38} \mathrm{~km}$, for a DM-estimated distance of $0.38 d_{0.38} \mathrm{kpc}$ and a temperature of $61.7 \pm 3.0 \mathrm{eV}$. Emission from the compact nebula is well described by an absorbed power law model with a photon index of $\Gamma=1.67 \pm 0.06$, while the diffuse emission seen as a trail extending northeast of the pulsar shows no evidence of synchrotron cooling. We also applied image deconvolution techniques to search for small-scale structures in the immediate vicinity of the pulsar, but found no conclusive evidence for such structures.
\end{abstract}

Key words: pulsars: individual (PSR J1741-2054) - X-rays: general

\section{INTRODUCTION}

PSR J1741-2054 (J1741) is one of the closest middle-aged $\left(\tau_{c}=390 \mathrm{kyr}\right)$ pulsars known. It has a period of $P=413 \mathrm{~ms}$ and was first discovered in $\gamma$-rays using the Large Area Telescope (LAT) on the Fermi Gamma-ray Space Telescope by a blind search for periodic $\gamma$-ray pulsations from Fermi-LAT point sources (Abdo 2009). It was subsequently detected in archival Parkes radio data and observed using the Green Bank Telescope (Camilo et al. 2009). The pulsar has a spin-down energy loss rate of $\dot{E}=9.5 \times 10^{33} \mathrm{erg} \mathrm{s}^{-1}$ which is moderately low compared to those of other $\gamma$-ray pulsars. The pulsar has a very small dispersion measure $(\mathrm{DM})=4.7 \mathrm{pc} \mathrm{cm}^{-3}$ and a magnetic field of $2.7 \times 10^{12} \mathrm{G}$. Using the NE2001 Galactic electron density model, the low DM implies a distance of $0.38 \mathrm{kpc}$ (Cordes \& Lazio 2002). At this distance, its measured radio flux at $1400 \mathrm{MHz}(S \sim 160 \mu \mathrm{Jy})$ makes it one of the least luminous radio pulsars known. Its $\gamma$-ray pulsations lag behind its radio pulsations by $\delta=0.29 \mathrm{P}$, implying that our line of sight tangentially cuts the $\gamma$-ray cone, while nearly missing the radio beam (Camilo et al. 2009). This makes J1741 a transitional object between a classical radio/ $\gamma$-ray loud pulsar such as Vela and the radio-quiet Geminga-type pulsars. Interestingly, Romani et al. (2010) detected a $20^{\prime \prime}$ long, nonradiative $\mathrm{H} \alpha$ bow shock nebula around the pulsar. Modeling of the bow shock suggested that the pulsar is traveling with a velocity of $\sim 150 \mathrm{~km} \mathrm{~s}^{-1}$, while the observation of negative radial velocities from both sides of the nebula imply that the velocity is directed out of the plane of the sky at an angle of $15^{\circ} \pm 10^{\circ}$.
Using a short Chandra ACIS-S observation (observation ID (ObsID): 11251), Romani et al. (2010) detected an X-ray pulsar wind nebula (PWN) within this $\mathrm{H} \alpha$ nebula and a long (>2 arcmin) X-ray trail at an angle of $45^{\circ} \pm 5^{\circ}$ east from north. Romani et al. (2010) also suggested that there are asymmetries in the small scale structure surrounding the pulsar, which they associate with a compact 2!!5 equatorial toroidal structure. Marelli et al. (2014) and Karpova et al. (2014) performed a spectral analysis of the pulsar emission using $X M M-N e w t o n$ and Chandra and determined that a twocomponent (blackbody plus power-law) model is required to obtain satisfactory spectral fits.

In this paper, we use $\sim 300 \mathrm{ks}$ of Chandra data of J1741 that were obtained as part of the Cycle 14 Chandra Visionary Project "A Legacy Study of the Relativistic Shocks of PWNe," plus a $\sim 49 \mathrm{ks}$ archival observation, to constrain the pulsar motion, and the geometry of the PWN outflow. We discuss our approach to image registration and proper motion measurement in Section 3, followed by a discussion in Section 4 of our image deconvolution efforts to search for small-scale structure around the pulsar. In Section 5 we discuss the results of our spectral fits for the pulsar emission and that of the extended PWN, and compare these with previous results. In Section 6 we discuss the implications of the proper motion measurements, including comparisons with the observed $\mathrm{H} \alpha$ nebula surrounding J1741, and discuss the lack of evidence of for synchrotron cooling in the PWN trail. Our conclusions are summarized in Section 7. 
Table 1

Chandra Observations of PSR J1741-2054

\begin{tabular}{lcc}
\hline \hline Observation ID & Exposure Time (ks) & Observation Date \\
\hline Archival & $\ldots$ & $\ldots$ \\
11251 & 48.78 & $2010-05-21$ \\
New observations & $\ldots$ & $\ldots$ \\
14695 & 57.15 & $2013-02-06$ \\
14696 & 54.30 & $2013-02-19$ \\
15542 & 28.29 & $2013-04-01$ \\
15638 & 29.36 & $2013-04-02$ \\
15543 & 57.22 & $2013-05-15$ \\
15544 & 55.73 & $2013-07-09$ \\
\hline
\end{tabular}

\section{X-RAY DATA ANALYSIS}

We obtained $282 \mathrm{ks}$ of new Chandra ACIS-S exposure time of J1741. Table 1 lists the parameters for each of the six new observations that we obtained, including the archival observation. To reduce pileup, the CCDs were operated in half-frame VFAINT mode so that events were read out every $1.7 \mathrm{~s}$. The pulsar was placed near the optimum focus on the backside illuminated S3 chip. In addition to our six observations, there is a $48.8 \mathrm{ks}$ archival observation (ObsID: 11251), which was taken on 2010 May 21. Each new observation has a roll angle similar to the roll angle of the 2010 epoch $\left(\sim 90^{\circ}\right)$, except for ObsID: 15544 . This observation has a roll angle of $\sim 260^{\circ}$ as the nominal roll angle of Chandra is rotated by $180^{\circ}$ during the time of the year this observation was completed. The data were analysed with $C I A O 4.6 .2$ after all observations were reprocessed using the CALDB 4.5.9. No flaring occurred in any of the observations so the full exposure times were used.

Using all seven observations we produced a merged, exposure-corrected image of $\mathrm{J} 1741$ by reprojecting the new observations to a common tangent plane based on the WCS information of ObsID: 11251 (CIAO task: reproject_obs) and combined all reprojected observations into an exposure corrected image using the CIAO task flux obs. The merged ACIS-S image of the extended emission around the pulsar, smoothed with a $3^{\prime \prime}$ Gaussian, is shown in Figure 1. The pulsar point source lies at the apex of the diffuse X-ray emission, while a diffuse, faint $\mathrm{X}$-ray trail extending $\sim 1.9$ is seen toward the northeast of the pulsar.

\section{REGISTRATION AND THE PROPER MOTION}

To constrain the proper motion of the pulsar, we registered each of the new Chandra images to the archival image using field point sources that were identified using the $C I A O$ tool wavdetect. We selected sources with a detection significance of $>3 \sigma$ that were found on the S3 chip in both the 2010 observation and the corresponding new observation. These sources are highlighted in white and the pulsar is labeled as $P$ in Figure 1.

Careful consideration and modeling of the point-spread function (PSF) must be undertaken to reduce the effect of changes in the PSF shape on the count distribution of our point-sources. To improve the astrometric accuracy and reduce this effect, we simulate a PSF for each point source position, in each observation, and use it to fit for the position of the source.
To simulate a PSF of a point source, we use the software suite SAOTrace ${ }^{11}$ which is designed to simulate the propagation of photons from astronomical objects through the optics of the Chandra X-ray satellite. We use the aspect solution file of each observation and provided spectral information for the ray trace by extracting the spectrum of each stellar source using the CIAO task specextract. We model the spectra using an absorbed Mekal model in SHERPA. To improve the accuracy of the PSF modeling, we increase the normalization of this spectrum by a factor of 100 before passing it into SAOTrace. A model of a point source at its position is obtained by passing the raytrace from SAOTrace into the program MARX. ${ }^{22}$ Each PSF model is corrected for the science instrument module (SIM) offset from nominal location and filtered using the Good Time Interval data from the original event file.

To determine the position of the sources we use the maximum likelihood "figure of merit" (FoM) technique developed by van Etten et al. (2012). We generate a 39 pixel by 39 pixel image of the modeled PSF, binned to 1/9 ACIS pixel resolution. This is then compared to $0.3-5.0 \mathrm{keV}$ source images of the same size but binned to native ACIS pixel resolution. PSF models and source images are produced for all observations and for each registration source. The PSF is shifted along the $x$ and $y$ axes of the 1/9 pixel grid and rebinned to native ACIS pixels. We then compute the FoM at each offset in pixel coordinates, giving us a map of the likelihood of the observed counts with respect to the $x$ and $y$ position. To determine the best-fit position of the source, we fit a twodimensional Gaussian to the FoM surface, with the minimum of this surface providing the best fit position of the source. The standard deviations along $x$ and $y$ are estimated by calculating the square root of the eigenvalues of the covariance matrix.

Prior to registration we checked each source for any optical counterparts using VizieR. ${ }^{13}$ Sources $6,8,9,10$ and 13 all have optical counterparts within $2^{\prime \prime}$ of the PSF-fit position. Source 8 and 13 have estimated proper motions of $\left(\mu_{\text {R.A. }}, \mu_{\text {Decl. }}\right)=(22$,

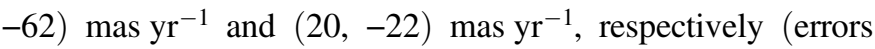

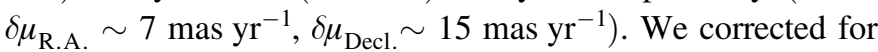
these nominal proper motions, but also confirmed that our final astrometric solution was insignificantly changed if we excluded these two stars from the analysis.

Using the FoM positions of our registration sources and their uncertainties as a reference grid to perform the relative astrometry, we determined the best translation transformation needed to register the images from the two epochs using the CIAO command wcs_match. For each observation we adopt the Chandra-determined roll angle. In Table 2 we list the bestfit frame shifts and their uncertainties. The uncertainties in these shifts were calculated by adding in quadrature the errors in the differences between each source before and after shift. Adding a rotation to the transformation did not produce a statistically significant improvement on the best-fit translation.

To calculate the position of the pulsar after registration, we first calculate the position of the pulsar in the unregistered frames. We simulate a PSF at the position of the pulsar in each observation using the method described for the registration sources. To define the energy dependence of the PSF, we extract a spectrum of the pulsar and fit it with an absorbed

\footnotetext{
11 http://cxcoptics.cfa.harvard.edu/SAOTrace/Index.html

$12 \mathrm{http} / / /$ space.mit.edu/CXC/MARX/

$13 \mathrm{http} / / /$ vizier.u-strasbg.fr/viz-bin/VizieR
} 


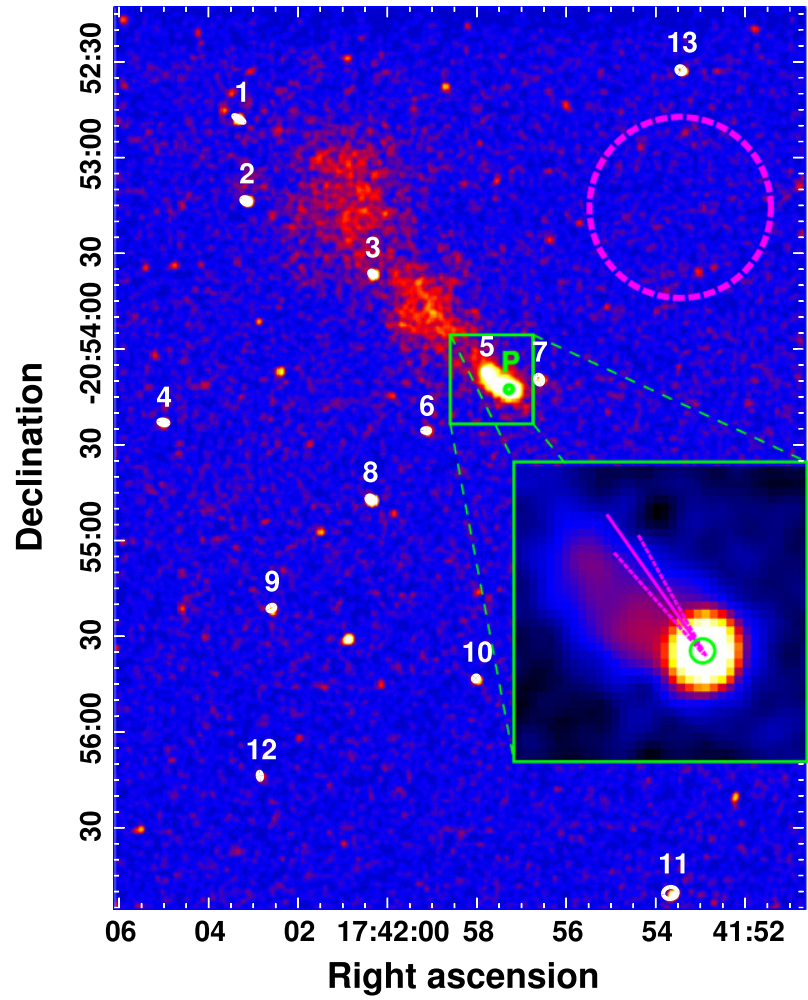

Figure 1. Merged Chandra exposure-corrected 0.3-5.0 keV ACIS-S image of the extended emission around J1741-2051. This was produced using reproject obs and flux obs and incorporates all available Chandra observations. The image is smoothed using a Gaussian of width $3^{\prime \prime}$ and plotted on the logarithmic scale. The reference sources numbered 1 through 13 were used for relative astrometry, while the pulsar is labeled as $P$. Other sources seen in the image are not suitable for astrometry because, being variable, they were not significantly detected in both the archival observation and one of the new epoch observations. We included a cutout region around the pulsar to show the point source. The magenta lines show our derived proper motion (solid) and its uncertainty (dashed), traced back from the pulsar position. The background region used for spectral analysis is shown as the magenta dashed circle.

Table 2

Frame Shifts and Their Uncertainties Used for Registration

\begin{tabular}{lrr}
\hline \hline ObsID & \multicolumn{1}{c}{$\begin{array}{c}\text { R.A. } \\
(\operatorname{arcsec})\end{array}$} & \multicolumn{1}{c}{$\begin{array}{c}\text { Decl. } \\
(\operatorname{arcsec})\end{array}$} \\
\hline 14695 & $-0.02 \pm 0.02$ & $0.48 \pm 0.02$ \\
14696 & $-0.01 \pm 0.02$ & $0.25 \pm 0.02$ \\
15542 & $0.02 \pm 0.04$ & $0.43 \pm 0.04$ \\
15638 & $-0.06 \pm 0.03$ & $0.36 \pm 0.03$ \\
15543 & $0.23 \pm 0.02$ & $0.29 \pm 0.03$ \\
15544 & $-0.11 \pm 0.03$ & $-0.29 \pm 0.03$ \\
\hline
\end{tabular}

power law plus blackbody model (see Section 5). For each observation, we generate a 6 pixel by 6 pixel image of the PSF model of the pulsar that is binned to $1 / 4$ ACIS pixel resolution. Using an image of the $0.3-5.0 \mathrm{keV}$ pulsar events of the same size and binning, we fit for the position of the pulsar in SHERPA. In this fit, we used a delta function (the pulsar) plus a two-dimensional Gaussian (the circumpulsar PWN), both convolved with the PSF. The resulting pulsar fit position for each frame was then registered by applying the best-fit transformations (Table 2).

To quantify the proper motion of the pulsar, we plot in Figure 2 the offset in the position of the pulsar between the archival observation and the new observations against the number of years since the archival observation. The uncertainties in the offset are calculated by adding in quadrature the uncertainties in the fit positions of the pulsar, the uncertainty in the frame shifts and the systematic uncertainty associated with choosing a particular tangent plane when creating an image in sky coordinates. We fit the offset using a linear function that corresponds to the positional shift of the pulsar between the archival observation and the new observations and this is seen as the dashed line in Figure 2.

We obtain a proper motion of $\mu_{\mathrm{R} . \mathrm{A} .} \cos (\delta)=-63 \pm 12$

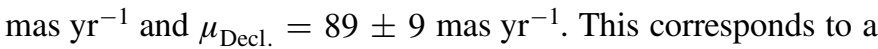

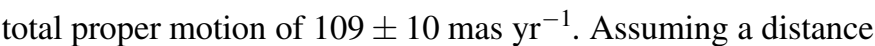
of $0.38 \mathrm{kpc}$ to the pulsar, this translates to a transverse velocity of $(196 \pm 18) d_{0.38} \mathrm{~km} \mathrm{~s}^{-1}$. The position angle of the proper motion is $215^{\circ} \pm 6^{\circ}$ east of north. The proper motion axis points in the opposite direction of the extended X-ray trail as expected (see Figure 1).

\section{X-RAY IMAGING}

Using the archival Chandra observation of J1741 (ObsID: 11251), Romani et al. (2010) performed a PSF subtraction of the pulsar point source to look for any small-scale structure surrounding the pulsar. They discovered that the region around the pulsar appears to be slightly extended and they associate this feature with the equatorial torus of the PWN. Using the same data as analyzed here, Karpova et al. (2014) searched for evidence of such structure by performing fits to a twodimensional Gaussian convolved with PSF models generated for each observation. They found no evidence for any smallscale extended features other than for a small emission feature associated with a known mirror artifact. ${ }^{14}$ We have carried out a similar investigation using image deconvolution techniques. We simulate a PSF of the pulsar in each observation using MARX following re-reduction of our Chandra observations with CALDB 4.4.7 to match the calibration data used for MARX. We define the energy dependence of the PSF as described in Section 3 and use the dither pattern of the observation with an aspect blur of 0.07 , which corresponds to the uncertainty in the telescope pointing. ${ }^{15} \mathrm{We}$ also correct for SIM offset. Using this PSF we deconvolve a $0.3-5.0 \mathrm{keV}$ pulsar image using the Lucy-Richardson deconvolution algorithm (Lucy 1974) implemented using the CIAO task arestore. All images were binned to quarter-ACIS pixel. We ran arestore multiple times using a number of different iterations between 10 and 200 to determine convergence of new features. No new structures appeared after 50 iterations.

The deconvolved image from one observation is shown in Figure 3 . The emission is well described by a point source, with the exception of the artifact feature (identified in cyan). We thus see no conclusive evidence of other small-scale structure in the immediate region surrounding the pulsar, consistent with the results reported by Karpova et al. (2014).

\footnotetext{
14 http://cxc.harvard.edu/ciao/caveats/psf_artifact.html

15 http://space.mit.edu/CXC/marx/news.html
} 

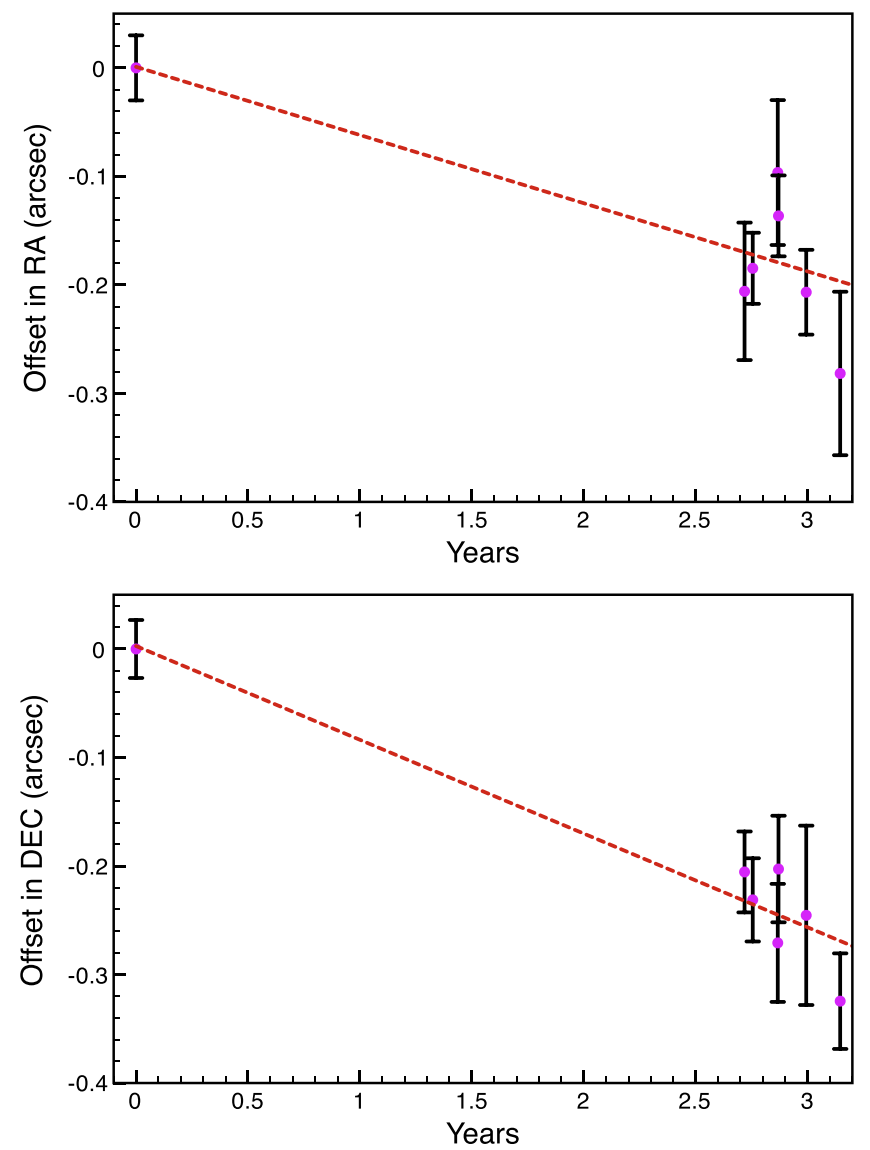

Figure 2. Offset in the position of the pulsar in R.A. (top) and decl. (bottom) in the new observations from the position of the pulsar in the archival observation plotted against the time since the first observation of PSR J1741 with Chandra (years). The dashed line corresponds to the line of best fit in which the slope corresponds to the proper motion of the pulsar.

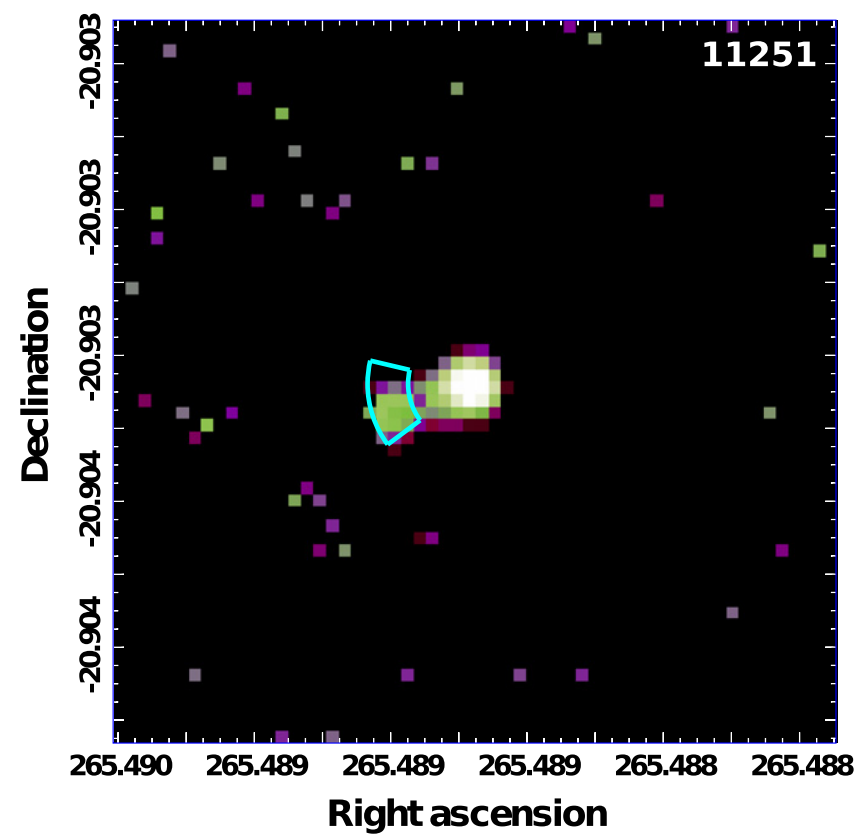

Figure 3. Deconvolved image of J1741-2054 from ObsID 11251 obtained using arestore after 50 iterations. The cyan region corresponds to the PSF asymmetry seen in Chandra data when pushing to sub-ACIS-pixel resolution. The image has been logarithmically stretched and the color enhanced to highlight the observed features.

\section{SPECTRAL ANALYSIS OF THE X-RAY EMISSION OF THE PULSAR AND ITS TRAIL}

\subsection{Pulsar}

As noted above, modeling of the Chandra PSF for use in determining an accurate pulsar position requires knowledge of the source spectrum. XMM-Newton observations establish a two-component spectrum for the pulsar, with a blackbody accompanied by a power law (Marelli et al. 2014). Similar results were derived by Karpova et al. (2014) using the same Chandra data reported here. We have re-analyzed these data by extracting events from a 0.70 radius region centered on the pulsar, shown as the green circle in Figure 4(a). The size of this region was chosen to minimise the contamination from the PWN, and subsequent modeling results have been corrected for the finite encircled energy fraction. All spectra were grouped with a minimum of 20 counts per bin, and a background spectrum was obtained using a source-free circular region with a radius of $30^{\prime \prime}$, as shown by the magenta circle in Figure 1. Using the CIAO pileup_map, we determined that approximately $5 \%$ of the pulsar events suffer from pileup. We thus included a pileup model in our spectral fits, where the frame time is $1.7 \mathrm{~s}$ and the PSF fraction is allowed to vary. All other parameters in the pileup model were frozen at default values.

To constrain the column density, we fit spectra from the compact nebula to the northeast of the pulsar (see Section 5.2) using an absorbed power law (more details in Section 5.2). We obtained $N_{H}=\left(1.20_{-0.07}^{+0.08}\right) \times 10^{21} \mathrm{~cm}^{-2}$, in good agreement with the above studies, and adopt this value in all our models of the pulsar spectrum. We use the Wilms et al. abundance table throughout our analysis (Wilms et al. 2000). Modeling the spectrum with a power-law plus blackbody model, we obtained a photon index of $\Gamma=2.68 \pm 0.04$ and a blackbody spectrum with a temperature of $k T_{\text {eff }}=61.7 \pm 3.0 \mathrm{eV}$, in excellent agreement with the results reported by Marelli et al. 2014 and Karpova et al. (2014). Omitting the pile-up correction yields similar values. We use the best-fit values above for PSF modeling of the pulsar.

We also ran fits using magnetized neutron star atmosphere models (nsa and nsmax in SHERPA) for the thermal component. These gave somewhat different temperatures and emitting areas, but did not significantly improve the quality of the fit. For example a magnetic carbon atmosphere model (nsmax model 12006, Mori \& Ho (2007)) gave a temperature of $k T_{\text {eff }}=86.0 \pm 9.0 \mathrm{eV}$ and emission radius of $R_{\text {emis }}=\left(4.90_{-2.3}^{+3.0}\right) d_{0.38} \mathrm{~km}$. The power law component was only slightly affected with $\Gamma=2.63 \pm 0.03$.

\section{2. $P W N$ and its Extended $X$-ray Trail}

To analyse the spectrum of the compact nebula described above, we extracted spectra from each observation using the cyan rectangular region in Figure 4(a) and combined these using specextract. We used the same background spectrum as for the pulsar spectrum. The compact nebula contains a total of $\sim 900$ counts and we binned the combined spectrum with a minimum of 20 counts per spectral bin. The PWN spectrum is consistent with an absorbed power law with an index of $\Gamma=1.60 \pm 0.20$.

To determine whether there is any spectral variation in the PWN and its extended trail, we extract spectra from the five regions defined in Figure 4(b). These regions correspond to roughly the same spectral regions reported by Marelli et al. 

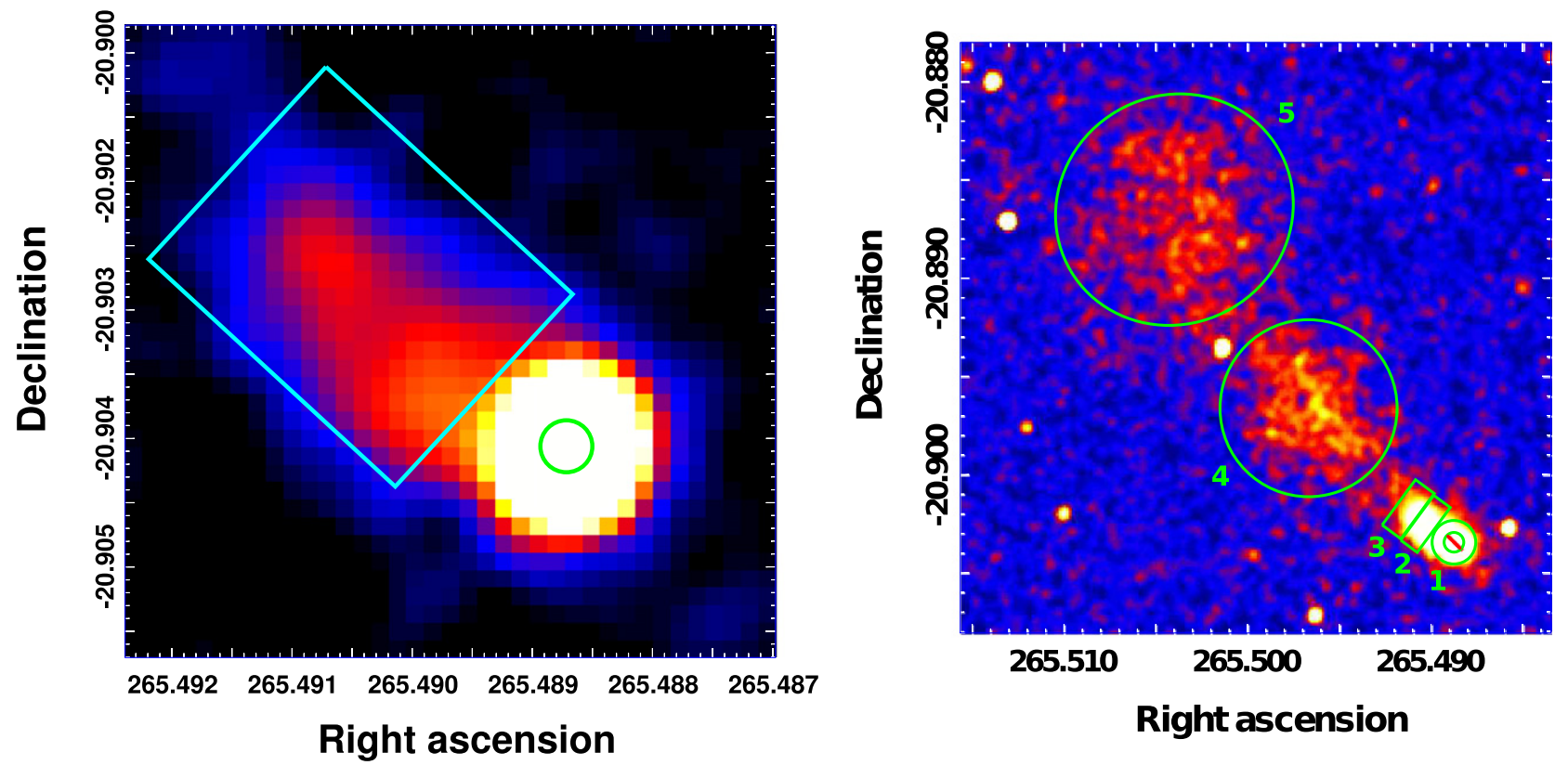

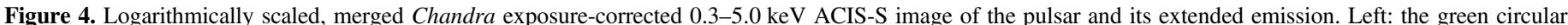

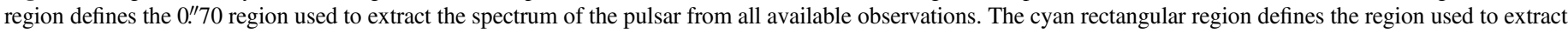

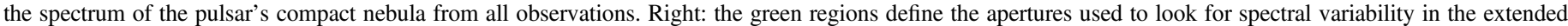
emission of the pulsar. The pulsar was excluded from the spectral extraction of region 1 .

Table 3

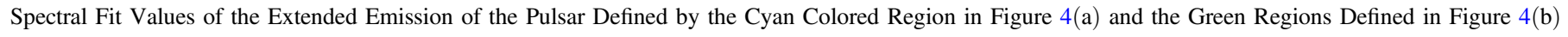

\begin{tabular}{|c|c|c|c|c|c|}
\hline Region & $N_{H}\left(\times 10^{21}\right) \mathrm{cm}^{-2 \mathrm{a}}$ & $\Gamma$ & $\begin{array}{c}\text { Absorbed Flux } \\
10^{-14} \mathrm{erg} \mathrm{cm}^{-2} \mathrm{~s}^{-1}\end{array}$ & $\begin{array}{l}\text { Unabsorbed Flux } \\
10^{-14} \mathrm{erg} \mathrm{cm}^{-2} \mathrm{~s}^{-1}\end{array}$ & $\chi^{2} /$ dof \\
\hline Compact PWN & $1.20_{-0.07}^{+0.08}$ & $1.60 \pm 0.20$ & $2.86_{-0.20}^{+0.17}$ & $3.15_{-0.07}^{+0.09}$ & 0.90 \\
\hline 1 & $\ldots$ & $1.97_{-0.17}^{+0.18}$ & $1.56_{-0.12}^{+0.13}$ & $1.81 \pm 0.33$ & 0.99 \\
\hline 2 & $\ldots$ & $1.50_{-0.15}^{+0.16}$ & $1.54_{-0.10}^{+0.15}$ & $1.67 \pm 0.16$ & 0.84 \\
\hline 3 & $\ldots$ & $1.57_{-0.19}^{+0.20}$ & $0.81_{-0.11}^{+0.08}$ & $1.10 \pm 0.11$ & 0.80 \\
\hline 4 & $\ldots$ & $1.63_{-0.11}^{+0.12}$ & $4.35_{-0.15}^{+0.28}$ & $4.81_{-0.14}^{+0.04}$ & 0.99 \\
\hline 5 & $\cdots$ & $1.70_{-0.11}^{+0.10}$ & $5.40_{-0.36}^{+0.29}$ & $6.03_{-0.16}^{+0.06}$ & 1.16 \\
\hline
\end{tabular}

Note. All uncertainties are one $\sigma$.

${ }^{\mathrm{a}}$ Fixed at value from joint compact nebula.

(2014), except that we investigate smaller regions in the compact nebula near the pulsar. In the outer portions of the nebula, the count rate is too low to obtain good spectra in smaller regions. We model each region individually using an absorbed power law, where we fix column density to the value derived earlier from fits to the inner nebula region but let photon index and the normalization vary. We have listed in Table 3 the absorbed flux, unabsorbed flux and the reduced $\chi^{2}$ for each region, as well as the best-fit parameters from fitting the trail spectra. There is no evidence of systematic variation in the photon index of the compact nebula and trail (regions 1-5). The photon index of region 1 is slightly higher than that of the other four regions, but is consistent within uncertainties with all regions except for region 2 . This slight variation between region 1 and 2 could suggest that region 1 is affected by leakage of the softer emission from the point source. Modeling the photon index as a function of distance from the pulsar using a linear regression fit with a constant function in SHERPA, we obtain $\Gamma=1.67 \pm 0.06$ for the trail. The global PWN index derived from the SHERPA fit and the values we obtained in Table 3 are consistent with Karpova et al. (2014) and Marelli et al. (2014), who derived $\Gamma=1.74 \pm 0.07$ and $\Gamma=1.78 \pm 0.15$ respectively for the PWN.

\section{DISCUSSION}

Using Chandra observations of PSR J1741-2054 that span a $\sim 3.2$ yr period, relative astrometry measurements have identi-

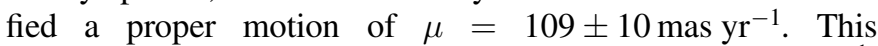
corresponds to a modest velocity of $(196 \pm 18) d_{0.38} \mathrm{~km} \mathrm{~s}^{-1}$, which agrees well with the velocity derived by Romani et al. (2010) using $\mathrm{H} \alpha$ spectroscopy. The larger distance of Karpova et al. (2014) gives a transverse velocity of $\sim 400 \mathrm{~km} \mathrm{~s}^{-1}$, inconsistent with that obtained from optical spectroscopy. The direction of the proper motion is $205^{\circ} \pm 6^{\circ}$ east of north, opposite the elongated X-ray trail. Ng \& Romani (2004); Johnston et al. (2005) and Ng \& Romani (2007) found that the direction of proper motion of a pulsar is, generally, approximately parallel to its rotation axis.

In Figure 5 we plot the pulsar track (red) in Galactic coordinates (note the expanded $b$ scale). This has the pulsar skimming above the plane. It does not intersect the plane itself unless one extrapolates an unreasonable $\sim 2 \times 10^{6} \mathrm{yr}$; 


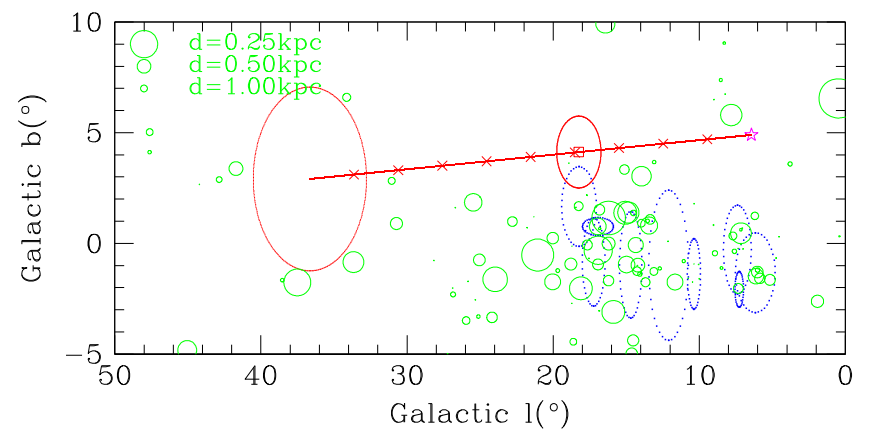

Figure 5. The past trajectory (red) of PSR J1741-2051 in Galactic coordinates. Positions are marked every $10^{5} \mathrm{yr}$, with uncertainty ellipses at $\tau_{c}=3.91 \times 10^{5}$ $\mathrm{yr}$ and at $10^{6} \mathrm{yr}$. For comparison we show the locations and sizes of $\mathrm{OB}$ associations from the Mel'Nik \& Efremov (1995) catalog (blue dotted ellipses) and the Hipparcos sample of $\mathrm{OB}$ stars (green circles). A substantial concentration of $\mathrm{OB}$ stars at $\sim 0.3-0.6 \mathrm{kpc}$ lies near the pulsar track at $\sim \tau_{c}$ and a more distant $(d=1.45 \mathrm{kpc})$ OB association overlaps with the track uncertainty at this age.

however, the track starts within the $\sim 50$ pc OB star scale height for $t \sim \tau_{c}$ and distances $d<1 \mathrm{kpc}$. For comparison we plot the positions of the Hipparcos catalog OB stars (green), with circle size proportional to the parallax. This set is quite complete, with useful parallaxes, to $\sim 500 \mathrm{pc}$, and increasingly incomplete at larger distances. At large distance the cataloged OB associations (Mel'Nik \& Efremov 1995) provide plausible pulsar birthsites, and their cataloged extent is plotted by the blue dotted ellipses. Intriguingly, one association overlaps the pulsar track, but this is at a likely unreasonable catalog distance of $1.45 \mathrm{kpc}$. We conclude that, with the pulsar motion passing along the Galactic plane, there will be many superposed massive star locations, and no definitive birthsite can be identified. However, there are certainly many plausibly associated massive stars consistent with our preferred $d \sim 0.4 \mathrm{kpc}$, especially considering that some pulsar progenitors may be $\mathrm{OB}$ runaways with significant offset during their pre-explosion lifetime.

Neither our deconvolved nor our PSF-subtracted images indicate conclusive evidence of small-scale structure surrounding the pulsar that might be associated with a torus or jet-like feature. The equatorial torus structure that Romani et al. (2010) associate with a diagonal excess seen $\sim 0$.'75 from the core of the pulsar image seems to have arisen from the mirror asymmetry. Karpova et al. (2014) perform a similar analysis and come to the same conclusion. However, it is interesting to compare the nebula head and proper motion with the $\mathrm{H} \alpha$ structure described in Romani et al. (2010). In Figure 6 we see that the pulsar lies very close to the bow shock limb (accuracy limited by our relative X-ray/optical astrometry). Interestingly, our measured proper motion is consistent with, although nominally slightly south of, the $\mathrm{H} \alpha$ nebula's symmetry axis. However the X-ray PWN trail fills only the southern half of the apparent $\mathrm{H} \alpha$ cavity, punching out through a gap at the back end of the $\mathrm{H} \alpha$ emission and continuing to the arcmin-scale trail beyond. The origin of this asymmetry is unclear, but a clue may be seen in the X-ray contours, whose ridge line lies at PA $\approx 70^{\circ}$, i.e., misaligned with the proper motion by $\approx 35^{\circ}$. This suggests a second symmetry axis in the PWN, possibly due to a pulsar jet or other outflow concentration. This directs the shocked PWN plasma to the southeast, preferentially filling this half of the $\mathrm{H} \alpha$ cavity. A more complete discussion of the PWN geometry, including the $3 \mathrm{D} \mathrm{H} \alpha$ kinematics, is in preparation.
The X-ray spectrum of the pulsar requires a combination of non-thermal and thermal model components. The emission is dominated by the non-thermal component ( $\sim 75 \%$ of unabsorbed flux), indicating that the majority of the X-ray emission is magnetospheric in nature. The emitting radius implied by the blackbody model for $\mathrm{J} 1741$ corresponds to $\left(4.5_{-2.5}^{+3.2}\right) d_{0.38} \mathrm{~km}$. This is substantially smaller than any viable neutron star radius (Lattimer \& Prakash 2007), suggesting that this thermal emission arises from hot spots on the surface, plausibly near the magnetic poles (Ho \& Heinke 2009). In fact, Marelli et al. (2014) do detect a pulsed thermal component for PSR J1741, also supporting such a surface temperature inhomogeneity.

The X-ray emission from the compact nebula and the trail is consistent with an absorbed power law. There is no discernible evidence of spectral variation with distance from the pulsar and the spectrum of the entire tail can be described by $\Gamma=1.67 \pm 0.06$. We compute the minimum (equipartition) energy by approximating the X-ray emission from the PWN (region 2, 3, 4 and 5 in Figure 4(b)) as a cylinder with length $l \sim 108 \operatorname{arcsec}\left(0.20 d_{0.38} \mathrm{pc}\right)$ and width $w \sim 18 \operatorname{arcsec}$ $\left(0.03 d_{0.38} \mathrm{pc}\right)$, comprising a volume of $V \sim 5.0 \times 10^{51} \phi d_{0.38}^{3}$ $\mathrm{cm}^{3}$, where $\phi$ is filling factor. The minimum energy in relativistic particles and magnetic field required to produce a synchrotron source of a given luminosity (Pacholczyk 1970) yield $E_{\min } \sim C(1+\kappa)^{4 / 7} V^{3 / 7} L_{\mathrm{syn}}^{4 / 7}$, where $\kappa$ is the ion to electron energy ratio, $L_{\text {syn }}$ is the synchrotron luminosity and $C$ is a function dependent on energy, electron charge, speed of light and the mass of the electron in Gaussian cgs units (see Pacholczyk 1970). In the following, we considering only the leptonic case, where $\kappa=0$. The total luminosity of the PWN is $L(0.5-10.0 \mathrm{keV})=2.36 \times 10^{30} \mathrm{erg} \mathrm{s}^{-1}, \quad$ giving $E_{\min } \sim 5.50 \times 10^{40} \phi^{3 / 7} d_{0.38}^{17 / 7} \mathrm{erg}$. The associated minimumenergy magnetic field is $B_{\min } \sim\left(D(1+\kappa) L_{\text {syn }}\right)^{2 / 7} V^{-2 / 7}$, where $D$ is a function similar to $C$. This magnetic field is $\sim 15 \phi^{-2 / 7} d_{0.38}^{-2 / 7} \mu \mathrm{G}$, leading to a lifetime of the X-ray emitting leptons of $\tau_{\mathrm{syn}} \sim 6.4 \times 10^{4} B_{\mu \mathrm{G}}^{3 / 2} E_{\mathrm{keV}}^{-1 / 2} \mathrm{yr}$ or $\sim 1100 \mathrm{yr}$ at an observed photon energy of $1 \mathrm{keV}$. This is comparable to the (length/proper motion) $=108^{\prime \prime} / 109 \sim 10^{3} \mathrm{yr}$ required for the pulsar to traverse the bright trail with our observed proper motion. Thus it is not surprising that there is no dramatic spectral steeping along the trail. If the PWN electrons flow at even faster speeds within the trail, this conclusion is even stronger.

\section{CONCLUSION}

Using $\sim 300 \mathrm{ks}$ of Chandra ACIS-S observations of PSR J1741-2054, we were able to determine the proper motion of the pulsar with a detection significance $>3 \sigma$. The direction of the proper motion is aligned with the extended PWN emission, and corresponds well with a symmetry axis of the associated $\mathrm{H} \alpha$ nebula. The diffuse X-ray emission immediately behind the pulsar is concentrated in the southeastern portion of the $\mathrm{H} \alpha$ nebula, possibly suggesting another flow axis from a jet or torus in the pulsar system. The trajectory of the pulsar, extrapolated over the characteristic age, does not provide a compelling correlation with known OB associations at the distance of the pulsar, although there are many massive stars consistent with this distance that could potentially have had a common origin.

The pulsar spectrum is well described by an absorbed power law accompanied by a blackbody with an emission radius of 


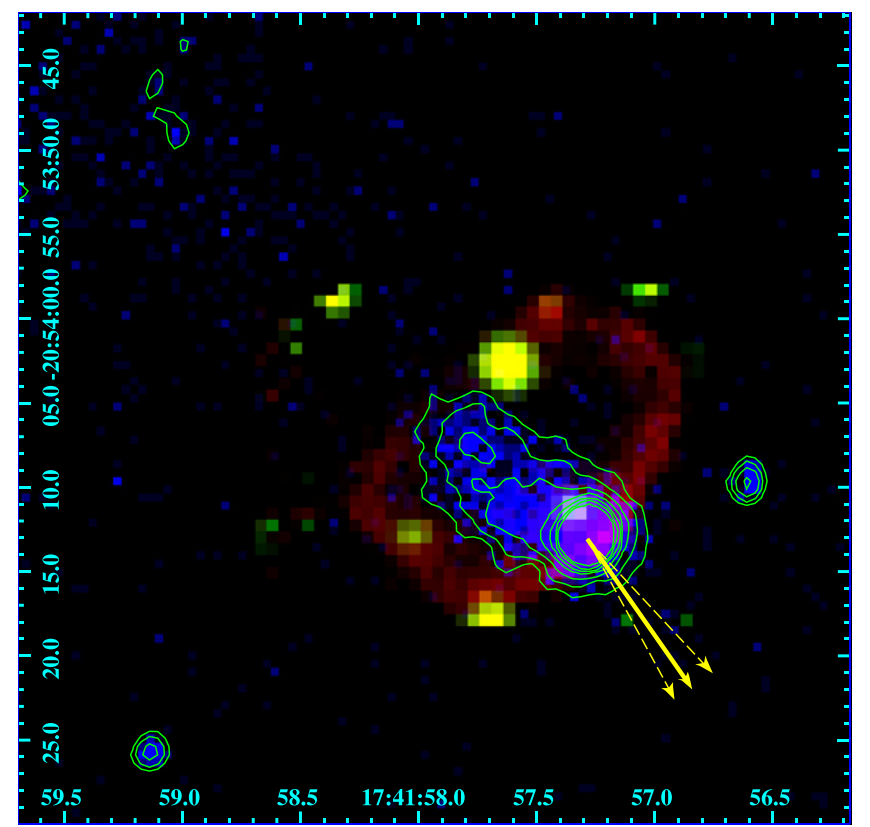

Figure 6. The head of the PSR J1741-2051 nebula. The red channel shows Ho emission at 6562-6564 $\AA$ while the green channel shows the average of the continuum $6551-6561,6565-6575 \AA$ with data abstracted from an AAT SPIRAL IFU observation. The blue channel shows the $0.3-7 \mathrm{keV}$ X-ray photons from our combined new observation. Green contours help one to read the X-ray structure, while the yellow arrows show our measured proper motion, with the arrow heads marking the position in $100 \mathrm{yr}$.

$\left(4.5_{-2.5}^{+3.2}\right) d_{0.38} \mathrm{~km}$ and a temperature of $k T_{\mathrm{eff}}=61.7 \pm 3.0 \mathrm{eV}$, as found in earlier works. The thermal component, a hot region on the neutron star surface, is augmented by a magnetospheric or unresolved PWN power law component. The PWN plus its extended trail can be well described using an absorbed power law and there is no evidence of variation in the photon index with distance from the pulsar. The integrated luminosity of the PWN over the $0.5-10 \mathrm{keV}$ is $2.36 \times 10^{30} \mathrm{erg} \mathrm{s}^{-1}$. This represents $0.02 \%$ of the pulsar spin down power, which is not atypical. We find no conclusive evidence of small-scale structure surrounding the pulsar that we can associate with a torus or jet-like structure.

\section{REFERENCES}

Abdo, A. A. e. a. 2009, Sci, 325, 840

Camilo, F., Ray, P. S., Ransom, S. M., et al. 2009, ApJ, 705, 1 Cordes, J. M., \& Lazio, T. J. W. 2002, arXiv:astro-ph/0207156

Ho, W. C. G., \& Heinke, C. O. 2009, Natur, 462, 71

Johnston, S., Hobbs, G., Vigeland, S., et al. 2005, MNRAS, 364, 1397

Karpova, A., Danilenko, A., Shibanov, Y., Shternin, P., \& Zyuzin, D. 2014, ApJ, 789, 97

Lattimer, J. M., \& Prakash, M. 2007, PhR, 442, 109

Lucy, L. B. 1974, AJ, 79, 745

Marelli, M., Belfiore, A., Saz Parkinson, P., et al. 2014, ApJ, 790, 51

Mel'Nik, A. M., \& Efremov, Y. N. 1995, AstL, 21, 10

Mori, K., \& Ho, W. C. G. 2007, MNRAS, 377, 905

Ng, C.-Y., \& Romani, R. W. 2004, ApJ, 601, 479

Ng, C.-Y., \& Romani, R. W. 2007, ApJ, 660, 1357

Pacholczyk, A. G. 1970, Radio Astrophysics. Nonthermal Processes in Galactic and Extragalatic Sources, ed. G. Burbidge, \& M. Burbidge (San Francisco, CA: Freeman)

Romani, R. W., Shaw, M. S., Camilo, F., Cotter, G., \& Sivakoff, G. R. 2010, ApJ, 724, 908

van Etten, A., Romani, R. W., \& Ng, C.-Y. 2012, ApJ, 755, 151

Wilms, J., Allen, A., \& McCray, R. 2000, ApJ, 542, 914 